REVISTA 92.1

Revista RELACIONES INTERNACIONALES

Escuela de Relaciones Internacionales.

Universidad Nacional, Costa Rica.

N. ${ }^{\circ} 92.1$ • Enero-Junio de 2019

doi: https://doi.org/10.15359/ri.92-1.3

Pp. $57-82$

\title{
LAS DECISIONES PÚBLICAS Y LAS ESTRATEGIAS DE DESARROLLO ANTE LA INCIDENCIA DE LOS ORGANISMOS INTERNACIONALES EN EL ESCENARIO DE CRISIS DE ESTADO EN MÉXICO ${ }^{1}$
}

\author{
PUBLIC DECISIONS AND DEVELOPMENT STRATEGIES IN THE \\ FACE OF THE IMPACT OF INTERNATIONAL ORGANIZATIONS \\ IN THE SCENARIO OF A STATE CRISIS IN MEXICO \\ Isaac Enríquez Pérez ${ }^{2}$ \\ ORCID 0000-0003-1361-5381
}

\section{RESUMEN:}

Las decisiones públicas y la intervención estatal en la dialéctica desarrollo/subdesarrollo, en buena medida, se definen y transforman a la luz de la gravitación que ejercen los organismos internacionales y los espacios globales para la toma de decisiones; especialmente a partir del despliegue de su poder epistémico/cognitivo que articula comunidades epistémicas transterritoriales a las cuales se incorporan activamente ministerios estratégicos y funcionarios especializados de las élites políticas nacionales.

La inserción de naciones subdesarrolladas como México no siempre es ventajosa, sino que se recrudece con la generalizada crisis de Estado y la pérdida de control respecto a las fuerzas, factores y poderes fácticos que condicionan la toma de decisiones. Se trata de reconocer que, entre las escalas nacionales donde se fraguan las decisiones públicas de las estrategias de desarrollo y el plano de los organismos internacionales, subyace una relación orgánica signada

\begin{abstract}
:
Public decisions and state intervention in the development/underdevelopment dialectic, to a large extent, are defined and transformed from of the gravitation exerted by international organizations and global spaces for decision-making; especially from of its epistemic/ cognitive power that articulates trans-territorial epistemic communities to which strategic ministries and high and middle specialized officials of national political elites are actively incorporated.
\end{abstract}

The insertion of underdeveloped nations such as Mexico is not always advantageous, but it is exacerbated by the generalized State crisis and the loss of control over the forces, factors and powers of fact that condition decision-making. It is about recognizing that between the national scales where the public decisions of the development strategies and the own plane of the international organisms are forged there lies an organic relationship

1 Este trabajo fue realizado en la Unidad Académica en Estudios del Desarrollo de la Universidad Autónoma de Zacatecas, y financiado por el Consejo Nacional de Ciencia y Tecnología (CONACyT) a través de su Programa de Estancias de Posdoctorales Vinculadas al Fortalecimiento de la Calidad del Posgrado Nacional.

2 Universidad Nacional Autónoma de México, Facultad de Ciencias Políticas y Sociales, México. Ph.D. en Economía del Desarrollo. Correo: isaacep@unam.mx 
por un condicionamiento recíproco y un consentimiento orientado a crear un discurso hegemónico, así como cursos de acción respecto al tratamiento de los problemas públicos y sus posibles soluciones. Enfatizar en el sentido y las facetas de esta relación estrecha es relevante porque contribuye a identificar las fuentes de las decisiones públicas. Para ello, se aprovechará el trabajo empírico realizado en otras investigaciones (principalmente el análisis de documentos estratégicos de algunos organismos internacionales) en aras de brindar contenido a conceptos que permitan comprender la acción social de estas organizaciones.

Palabras clave: dialéctica desarrollo/subdesarrollo, decisiones públicas, planeación del desarrollo, organismos internacionales, comunidades epistémicas trans-territoriales, crisis de Estado marked by a reciprocal conditioning and a consent oriented to create a hegemonic discourse, as well as courses of action regarding the treatment of public problems and their possible solutions. Emphasizing the meaning and facets of this close relationship is relevant because it helps identify the sources of public decisions. For this, the empirical work carried out in other researches (mainly the analysis of strategic documents of some international organizations) will be used in order to provide content to concepts that allow understanding the social action of these organizations.

Keywords: dialectic development/underdevelopment, public decisions, development planning, international organizations, transterritorial epistemic communities, State crisis

\section{Introducción}

Sin instituciones que condensen conocimientos, correlaciones de fuerzas y estructuras de poder, la dialéctica desarrollo/subdesarrollo tendería a profundizar sus contradicciones y el carácter destructivo de la lógica del mercado, signada por la anarquía y el afán de lucro y ganancia. Dentro de esos entramados institucionales que modelan, modulan y encauzan el comportamiento de la economía mundial y la política interestatal destacan los organismos internacionales y sus distintas funciones. Son estas instituciones las que -en sincronía con los gobiernos nacionales y en una lógica multidireccional-conforman una relación orgánica con condicionamientos recíprocos y dosis de consentimiento y compromiso orientados a perfilar una institucionalidad global.

Paralelamente a ello, una de las manifestaciones políticas del subdesarrollo en naciones que -como México- experimentan un declive de lo público y una endeble cohesión en su tejido social, es la crisis de Estado manifestada en la fragilidad de sus instituciones y en un predominio de la ilegalidad, la trasgresión constante de la ley y la impunidad. Estos últimos fenómenos -que parecen estrictamente endógenos y gestados a la luz de poderes fácticos locales/nacionales- se conjugan con la gravitación que ejercen los espacios globales para la toma de decisiones en aras de perfilar en la escala mundial una armonización, estandarización, homogeneización, convergencia y coordinación de las políticas públicas.

Reconocidas estas tendencias, cabe preguntarse lo siguiente: ¿Cuál es la incidencia que ejercen los organismos internacionales en las decisiones públi- 
cas y en el diseño de las estrategias de desarrollo propias de naciones periféricas como México? ¿Qué papel desempeña el poder epistémico/cognitivo de estas organizaciones en el proceso de planeación? Estas preguntas responden a una tendencia más amplia relacionada con la configuración de una institucionalidad global en la cual participan múltiples fuerzas, factores y agentes nacionales y transnacionales que tienden a entrelazarse y a conformar comunidades epistémicas a partir de la relación orgánica entre las élites políticas nacionales y los funcionariados internacionales.

Esbozadas estas interrogantes, cabe señalar que el objetivo general del presente texto consiste en desentrañar y analizar la intergénesis y las relaciones orgánicas entre los espacios globales para la toma de decisiones -especialmente los organismos internacionales-y las entidades nacionales dedicadas al proceso de planeación y al diseño de estrategias de desarrollo, en un contexto de declive de la vida pública y de intensificación de la crisis de Estado en México. Esto significa analizar, en tanto objeto de estudio, el sentido de las decisiones públicas que atienden la dialéctica desarrollo/subdesarrollo y que se vertebran a partir del eslabonamiento de las directrices y estrategias esbozadas por los organismos internacionales y las decisiones tomadas en las escalas nacionales en torno a los problemas públicos. Para cumplir con este objetivo de investigación, será aprovechado el trabajo empírico realizado en otros estudios propios (principalmente el análisis de las concepciones sobre el desarrollo y de documentos estratégicos difundidos desde algunos organismos internacionales como el Banco Mundial) en aras de dotar de contenido los conceptos que contribuyan a comprender las facetas y la acción social de estas organizaciones interestatales.

Una tesis fundamental del estudio que está detrás del presente documento fue la siguiente: entre los organismos internacionales y las élites político/tecnocráticas nacionales se presenta un condicionamiento recíproco y una simbiosis articulada por el poder epistémico/cognitivo que despliegan los primeros en la toma de decisiones públicas, así como en la configuración de las estrategias de desarrollo. Sin embargo, en sociedades subdesarrolladas como la mexicana -si bien no existe una imposición unidireccional-, el terreno se torna desnivelado ante circunstancias signadas por la fragilidad institucional y la crisis de Estado delineada a partir de la incidencia de múltiples poderes fácticos. De ahí la relevancia de adoptar un enfoque de teoría social crítica que contribuya a desentrañar la naturaleza de la reconfiguración del orden mundial y el posicionamiento de agentes transnacionales en el curso de la economía mundial y de la política internacional. Al identificar, para ello, estructuras históricas -concepto adoptado por Cox, 1981- que cohesionan fuerzas como las capacidades materiales, las ideas y las instituciones. 


\section{El poder epistémico/cognitivo de los organismos internacionales y la formación de comunidades epistémicas transterritoriales}

Los organismos internacionales son estructuras institucionales y organizacionales que sintetizan una hegemonía mundial y que derivan de asociaciones voluntarias y negociaciones entre Estados que ceden porciones de su soberanía para atender - desde la acción colectiva-tópicos propios de la economía mundial y la política internacional, así como los problemas públicos compartidos y comunes entre las naciones subdesarrolladas. Si bien esa noción es común en los debates propios de los estudios relativos a las relaciones internacionales (por ejemplo Cox, 1981, 1983 y 1987; Cox and Schechter, 2002; Keohane, 1989 y 1998), cabe matizar que, a su vez, estas estructuras se articulan como sistemas epistémicos, ideológicos, técnicos y financieros. Como tales condensan relaciones de poder, modelos de sociedad y mecanismos de gestión y regulación de las relaciones económicas y políticas internacionales; al tiempo que inciden en los cauces mismos que adopta la contradictoria y disruptiva dialéctica desarrollo/ subdesarrollo y el proceso de planeación en general (sobre el debate teórico y en torno al concepto y funciones de estas entidades véase Enríquez Pérez, 2017).

Ante la imposibilidad de los Estados y de las ciudadanías para erigir un gobierno mundial, los organismos internacionales contribuyen de manera decidida a configurar una mínima institucionalidad global que se apegue a los imperativos de la lógica que adopta el capitalismo como modo de producción y proceso civilizatorio cada vez más globalizado. En ello, desempeña un papel destacado la negociación, no siempre tersa, en el seno de los organismos interestatales; así como la creación, difusión y normalización de símbolos, normas, reglas y pautas de comportamiento sobre la base de la coordinación de esfuerzos intergubernamentales, la acción colectiva mundial, y la armonización, estandarización, homogeneización y sincronización de políticas públicas en distintos rubros nodales y estratégicos.

Esta institucionalidad global supone estructuras de poder y un posicionamiento diferenciado y asimétrico de los Estados, de cara a la definición y resolución de los problemas públicos, en aras de tornar funcional -que no suprimir- la desigualdad social e internacional experimentada en las sociedades subdesarrolladas. En esta correlación de fuerzas, la teledirección ejercida por los organismos internacionales adquiere relevancia al diseñar, desde las escalas nacionales, las estrategias de intervención para la posible solución de los problemas públicos. 
Estas organizaciones no son entidades aisladas ni tampoco son homogéneas a su interior - pues subyacen en ellas conflictos ideológicos que tensionan a sus miembros-, sino que forman parte - conjuntamente con otros actores y agentes hegemónicos- de una red global de toma de decisiones, en la cual sus funcionarios y consultores interactúan y se entrelazan con otros tomadores de decisiones y expertos a partir de la densidad de conocimientos, conceptos, identidades, códigos de comunicación y contactos políticos que les permiten pertenecer a grupos de poder compactos, autónomos y altamente cohesionados que hacen uso de la discrecionalidad, pese a tratarse de organizaciones financiadas con presupuestos estatales y que abordan tópicos y problemas públicos.

Lo que es posible observar es que estas comunidades epistémicas trans-territoriales $-\mathrm{y}$, particularmente, los organismos internacionales- se articulan a partir de un sistema epistémico/cognitivo que contribuye a la construcción de mecanismos de poder para la toma de decisiones a partir de la generación, tratamiento, procesamiento y manejo de información, conocimientos altamente especializados, sistemas conceptuales y datos estadísticos en torno a fenómenos locales y mundiales, y que se canalizan para intentar explicar y esclarecer $-\mathrm{y}$ en no pocas ocasiones y circunstancias a ensombrecer y encubrir- el comportamiento y problemáticas de las sociedades nacionales, la política internacional y la economía mundial; así como a configurar directrices normativas y cursos de acción que formen parte o se desprenden del diseño y ejercicio de estrategias de política pública.

Como se observa en organismos internacionales como el Fondo Monetario Internacional y el Banco Mundial (Enríquez Pérez, 2009, 2010, 2014, 2015 y 2016a:b), este poder epistémico se fundamenta en cuantiosos recursos humanos y presupuestales canalizados a la investigación y en los vastos cuerpos técnicos y altamente especializados que conforman centros de investigaciones al interior de estas organizaciones, y que tienen como una de sus finalidades generar documentos estratégicos y recomendaciones de política pública dirigidos a los funcionarios dedicados a la planeación dentro de los gobiernos nacionales y locales.

En una especie de división técnica del trabajo - de ahí la relevancia de la noción de comunidades epistémicas transterritoriales-, los organismos internacionales atienden distintas parcelas de la realidad social y contribuyen a definir los problemas públicos; a esbozar panoramas generales que le den forma y estructura al proceso de toma de decisiones seguido por los gobiernos; y a modelar el cauce de la dialéctica desarrollo/subdesarrollo. Mediante este poder epistémico, los organismos intergubernamentales eslabonan un discurso político/ideológico y técnico dotado de supuestos normativos (un deber ser) estructurados y regidos por principios, postulados y rasgos teóricos, éticos y empíricos, orientado a la construcción 
de una mínima base de legitimidad respecto a los objetivos, funciones e intervenciones de estas organizaciones y con relación al carácter polarizado, asimétrico y estratificado de las relaciones económicas y políticas internacionales.

A través de este discurso se configuran las redes y los espacios de toma de decisiones para coordinar la acción colectiva mundial; encauzar las deliberaciones y negociaciones diplomáticas; conciliar los intereses de los Estados partícipes; orientar el destino de asistencia financiera y técnica hacia las naciones que lo requieren; regir los procesos de planeación nacional; y para fundamentar las estrategias y concepciones sobre el desarrollo, delineadas en los documentos de política pública, diseñados y difundidos por los organismos internacionales.

Desde estas organizaciones interestatales se coordinan las acciones de cooperación internacional. En esos espacios globales para la toma de decisiones que se configuran, cada organismo interestatal participa en la deliberación y difusión de ideas en torno a temáticas y problemas que le dan forma a la agenda pública (Enríquez Pérez, 2015 y 2017; Valdez, 2015). No menos importante es su intervención en la formación de comunidades epistémicas (esta noción la desagrega Haas, 1992) desde su respectiva área de especialidad y con los expertos, técnicos y consultores que contribuyen al eslabonamiento de los sistemas epistémico/conceptuales que le dan forma a los códigos de comunicación y a las corrientes de pensamiento que hegemonizan el debate y la definición de los problemas públicos en las cumbres, paneles, foros, conferencias, reuniones anuales, convenciones, declaraciones, acuerdos y tratados, y que dan como resultado resoluciones, compromisos y documentos suscritos por los Estados miembros.

De ahí que se entreteja la mencionada división técnica del trabajo entre los organismos internacionales que participan en la red de manera diferenciada y con distintas dosis de poder y posición en la misma. Este poder está dado, en buena medida, por su discurso ideológico/político y los sistemas epistémico/conceptuales que perfilan la construcción social de la realidad modelan decisiones; estipulan cursos de acción y pautas de comportamiento; y definen estructuras institucionales que le otorgan sentido y legitimidad a la economía mundial, la política internacional, la reivindicación de los derechos humanos fundamentales, la dialéctica desarrollo/subdesarrollo y a las formas históricas de distribución de la riqueza.

Es de destacar que esta red de comunidades epistémicas transterritoriales está integrada por funcionarios nacionales de alto y mediano nivel radicados en ministerios y agencias, que controlan los trazos generales de la agenda pública (pensar, por ejemplo, en los bancos centrales, los ministerios de hacienda y economía) y que se incorporan a esos espacios globales para la toma de decisiones 
para interactuar en una relación orgánica con otros miembros de la red como el funcionariado y técnicos de los mismos organismos internacionales; consultores, asesores y académicos; directivos de Think Tank's privados, agencias calificadoras y cabilderos de intereses empresariales; activistas, líderes y técnicos de influyentes Organizaciones No Gubernamentales Internacionales (ONGI); y líderes de opinión. Todos ellos entrelazados a partir de intereses creados, compromisos, códigos de comunicación y conducta, ideologías, cosmovisiones y sistemas conceptuales compartidos que crean identidades grupales y fuertes mecanismos de cohesión y capital social.

Un ejemplo de lo anterior son las reuniones anuales del Grupo del Banco Mundial y el Fondo Monetario Internacional; el Comité para el Desarrollo promovido por ambos organismos; el Foro Económico de Davos; las conferencias ministeriales y las rondas de negociación de la Organización Mundial de Comercio. De eventos como estos se desprenden concepciones sobre el desarrollo, la pobreza, el crecimiento, que son compartidas por sus participantes; y ello es posible rastrearlo en los comunicados derivados de dichas reuniones.

A su vez, y al formar parte de las redes de los espacios globales para la toma de decisiones, los organismos internacionales contribuyen a la centralización y concentración del poder mundial y a tornar funcionales las desigualdades sociales e internacionales que son consustanciales al capitalismo. Además, estas entidades -a través de sus sistemas epistémico/cognitivos-conceptualizan e iluminan, bajo determinados supuestos y principios, ciertas aristas de la realidad social y de sus problemáticas, al tiempo que encubren otras facetas hasta el extremo de invisibilizarlas y omitirlas del espectro de los problemas públicos y sus respectivas agendas nacionales o mundiales. Estas concepciones, que relativamente homogenizan y estandarizan el pensamiento de las comunidades epistémicas transterritoriales y de las élites políticas que en las escalas nacionales hacen eco de sus códigos de comunicación, responden a las transformaciones históricas del capitalismo y a la correlación de fuerzas que priva al interior de estos organismos interestatales y de las estructuras geopolíticas y geoeconómicas.

A través de este poder epistémico/cognitivo -y de los supuestos normativos, éticos e ideológicos que le dan forma-, los organismos internacionales contribuyen al debate teórico propio de los estudios sobre el desarrollo; al tiempo que se inspiran en las teorías y metodologías provenientes de las llamadas universidades globales y esbozadas por académicos que en no pocos casos son asesores o consultores externos. 
Este pensamiento hegemónico no es neutral. Ello se evidencia en nociones como la de capacidades (Sen, 1999) que incide abiertamente en los índices, mediciones y concepciones del Programa de las Naciones Unidas para el Desarrollo (PNUD) y en la política social de naciones como México; o la de capital social (Putnam, 1993) y su acento en los proyectos autogestivos de las comunidades ante la retracción del Estado en materia de bienestar, y que es retomada en la agenda social del Banco Mundial; así como las versiones microeconómicas de las instituciones en el desempeño económico (Coase, 1984; Williamson, 1985, 1995, 1996 y 2000) que sustentaron la segunda generación de reformas promovida por el Banco Mundial (Burki y Perry, 1998).

No menos importante es su contribución a la acción de nombrar realidades y a categorizarlas en escalas de valores y prioridades mediadas por intereses creados; y a la creación de constelaciones de dominación y relaciones sociales ceñidas a ello. De ahí que sea posible sostener la premisa de que los actores socioeconómicos o políticos que expliquen e interpreten ciertas aristas del mundo fenoménico y que impongan sus saberes y discursos, lograrán apropiarse, dominar y controlar la realidad en torno a la cual construyen y difunden conocimiento e, incluso, pretenden delinear cursos de acción, construir mercados y definir las leyes sociales de la distribución de la riqueza.

De esta forma, los organismos internacionales hegemónicos (pensar por ejemplo, en el Banco Mundial) y las redes globales de toma de decisiones en las cuales sus funcionariados interactúan e intercambian ideas, conocimientos y saberes altamente especializados en torno a la agenda pública, conforman desde su fundación una diplomacia del desarrollo (concepto introducido por Babb, 2009) mediante los proyectos de cooperación internacional; las labores de asistencia técnica; y el despliegue de la gravitación y teledirección en torno al diseño y ejercicio de las políticas públicas y de las estrategias de desarrollo difundidas entre las naciones subdesarrolladas.

Lo que subyace en estos esfuerzos de acción colectiva global es la necesidad de crear regímenes internacionales (sobre esta noción véase Keohane, 1989). A través de estas estructuras jurídicas e institucionales se trata de brindar cauce y regulación a la estructura económica mundial y a las relaciones políticas entre Estados; para lo cual es prioritario promover la convergencia, sincronización, estandarización, armonización, homogeneización y coordinación de políticas públicas - particularmente de la política económica y de las demás agendas que guardan un vínculo de convergencia subordinada con sus concepciones ortodoxas y neoclásicas- en la escala planetaria. En ello es fundamental el poder epistémico/cognitivo y las funciones y labores de investigación respecto a los problemas públicos nacionales y mundiales; de deliberación teórica en torno 
a la dialéctica desarrollo/subdesarrollo; y de construcción de datos empírico/ estadísticos que orientan las decisiones públicas y el proceso de planeación de las naciones subdesarrolladas.

No menos importantes son los principios y pautas establecidos por estas agencias en torno a las funciones del Estado, sus transformaciones y demás instrumentos de intervención en el proceso económico, la configuración de los mecanismos de poder, la redistribución de la riqueza, y la procuración de derechos mínimos fundamentales que preserven la estabilidad sociopolítica indispensable para el proceso de valorización y acumulación del capital.

A grandes rasgos, no son los distintos instrumentos de financiamiento de proyectos los que mayor incidencia ejercen en el rumbo de las políticas públicas, sino que son las funciones y el poder epistémico/cognitivo de los organismos internacionales y de las comunidades epistémicas transterritoriales de las cuales forman parte, lo que en su conjunto perfila lenguajes, simbolismos, códigos de comunicación, concepciones sobre la realidad, ideologías, pautas de comportamiento, cursos de acción, entramados institucionales, estructuras organizacionales y mecanismos de intervención (consúltense estudios empíricos como Saxe-Fernández, 2003; Saxe-Fernández y Delgado-Ramos, 2004 y 2005; Corbalán, 2002; Toussaint, 2006; Enríquez Pérez, 2009, 2010, 2014, 2015 y 2016a:b). Lo anterior es fundamental en las capacidades de teledirección que se despliegan en la relación orgánica entre las agencias interestatales y los funcionariados nacionales dedicados a la planeación en ministerios y agencias gubernamentales, y que influyen en la vida diaria de poblaciones enteras. De ahí que la constelación de organismos internacionales es un escenario en el que convergen múltiples intereses y es disputado el control político e ideológico de las instituciones y de las leyes sociales en torno a la distribución de la riqueza en los planos nacional y mundial.

\section{La planeación del desarrollo a la luz de la relación orgánica en los espacios globales para la toma de decisiones}

Sin afán de incurrir en un determinismo ideológico, la intervención del Estado en los cauces de la dialéctica desarrollo/subdesarrollo a través del proceso de planeación y sus distintos instrumentos para modelar las sociedades, en una lógica bidireccional e interrelacionada, se corresponden con las directrices, supuestos y postulados de política pública confeccionados desde los organismos internacionales y desde los espacios globales para la toma de decisiones de los cuales, estos últimos, son agentes protagónicos. Entre las escalas nacionales de- 
dicadas a la definición y atención de los problemas públicos y los distintos mecanismos de acción colectiva mundial se suscita una relación orgánica dada por la simbiosis y sincronización de lenguajes, concepciones, pautas normativas y prácticas. En ello desempeña un relevante papel el poder epistémico/ideológico/técnico/financiero articulado desde las agencias multilaterales y desde las comunidades epistémicas transterritoriales, que es consentido, realimentado y asimilado por aquellos sectores del funcionariado nacional dedicado al diseño de las políticas de desarrollo.

Es ese sistema epistémico/conceptual emanado de las organizaciones interestatales y sus centros de investigación, lo que define ciertas directrices normativas y de política pública que orientan el pensamiento y la acción social de los funcionariados internacionales y los gobiernos nacionales. Estos lenguajes son, en esencia, selectivos, excluyentes, etnocéntricos y fungen como cohesionadores de otros discursos expuestos por distintos actores y agentes socioeconómicos y políticos -afines o antagónicos- que pretenden incidir en la confección de la agenda pública. Una excepción a esta tendencia la representaron los gobiernos progresistas sudamericanos que desde 1999 se distanciaron, al menos discursivamente, de la órbita de los organismos financieros internacionales.

Como ejemplo de lo anterior y como digresión respecto al mencionado poder epistémico/cognitivo y a la forzada agenda social de los organismos internacionales, podemos apuntar lo siguiente en el caso de la conceptualización tecnocrática de la pobreza: un pobre es tal no por la carencia de los medios básicos de subsistencia que abonan a la satisfacción de necesidades. Existe la pobreza porque una entidad con poder epistémico/cognitivo categoriza como tal a un grupo social que no se acerca al convencionalismo de lo que es "vivir bien" (o tener bienestar) en una sociedad y en un momento histórico determinados. Ese grupo social es concebido como objeto de asistencia con la finalidad de solventar la carencia de satisfactores y atender sus necesidades. Si la realidad de la pobreza se concibe y representa bajo criterios cuantitativistas y como la “incapacidad para alcanzar un nivel de vida mínimo" (World Bank, 1990:26 y 27); lo cual remite a una capacidad de consumo o de poder adquisitivo relativo a la nutrición; entonces las pautas normativas, las políticas públicas y los cursos de acción derivados de esa concepción estarán condicionados por ello. Y aunque se agreguen otras variables a la concepción de la pobreza -pensemos en la enfermedad, la carencia de acceso al agua potable y a servicios sanitarios y educativos, el desempleo, falta de vivienda, la exposición a la violencia y al crimen, la vulnerabilidad, la opresión de las libertades políticas-(International Monetary Fund and World Bank, 1999 y World Bank, 2001), el criterio cuantitativo del umbral de los dos dólares diarios se impone al momento de esbozar estrategias focalizadas para el tratamiento de un problema público. De ahí 
que políticas sociales neoasistencialistas como PROGRESA, Oportunidades o Prospera, adoptadas en México durante las últimas dos décadas, no cuestionen los fundamentos del Consenso de Washington que limitan la intervención del Estado en el proceso económico y en la redistribución de la riqueza; e, incluso, dichas políticas públicas son objeto de reconocimiento por parte de los mismos organismos internacionales (véase Levy, 2008; Banco Mundial, 2014).

En todo lo anterior, la ideología del desarrollo (sobre esta noción véase Sachs, 1992; Enríquez Pérez, 2015) funciona como un discurso cuyos objetivos son la construcción de mecanismos simbólicos de legitimación; la conciliación de intereses variados, dispersos y contradictorios presentes en el nivel nacional y en la comunidad internacional; así como el consenso o cooptación en los sistemas políticos nacionales. Además, pese a los conflictos ideológicos entre los organismos internacionales (sobre esta conflictividad véase Santiso, 2004; Nimiña y Larrarlde, 2018), los discursos emanados de los espacios globales para la toma de decisiones no se contraponen ni subvierten entre sí; más bien, se despliegan como parte del andamiaje que conforma los entramados institucionales, capaces de garantizar la expansión e integración global del capitalismo y la profundización de sus polarizantes mecanismos de acumulación.

La teledirección ejercida desde los espacios globales para la toma de decisiones respecto a los procesos de planeación en las sociedades nacionales es parte del ya mencionado imperativo de armonización, sincronización, homogeneización, estandarización, convergencia y coordinación de políticas públicas y que orienta la formación de una institucionalidad global. Desde los organismos internacionales se esbozan conceptos, directrices y pautas de comportamiento que definen debates públicos; se introducen nuevos tópicos y temas que abren y le dan forma a la agenda pública; y, a partir de ello, se plantean posibles soluciones y pautas normativas respecto a las problemáticas relacionadas con el crecimiento económico, los desequilibrios fiscales y monetarios, la pobreza, el deterioro del medio ambiente, las relaciones laborales, la salud pública, la política educativa, el comercio internacional, los derechos humanos, la gobernabilidad, entre otros (sobre algunos de estos temas concretos y la incidencia de los organismos internacionales véase los estudios de Sánchez Cerón, 2001; Santiso, 2001 y 2004; Delgado-Ramos y Saxe-Fernández, 2005; Reyes, 2015; Ascolani, 2008; Burgos Silva, 2009 y 2015; Barrera Cordero, 2009; Lerner, 2009; Picazzo Palencia, 2012; Fal, 2013; Noy, 2013; Enríquez Pérez, 2014 y 2016b).

Al conciliarse los intereses de las élites y funcionariados transnacionales y los propios de las tecnocracias nacionales no existe margen para argumentar que las directrices de política pública, sus supuestos y pautas normativas responden a una imposición discrecional, vertical y unidireccional desde los espacios 
globales para la toma de decisiones (este argumento de la imposición subyace en posturas como las expuestas por Saxe-Fernández, 2003; Saxe-Fernández y Delgado-Ramos, 2004 y 2005; Corbalán, 2002; Toussaint, 2006). Más bien y sin que exista una homogeneidad ideológica, lo que se presenta es una compenetración, así como un estrecho compromiso y simbiosis interesada entre los funcionariados nacionales e internacionales dedicados a la planeación y delimitación de la agenda pública, y los consultores externos, las élites económico/ financieras, las ONGI que, en su conjunto y como apuntamos arriba, se erigen como grupos de presión al hacer valer sus intereses creados.

Por ejemplo, las políticas de ajuste y cambio estructural regidas por la racionalidad del mercado y adoptadas desde la década de los ochenta en el mundo subdesarrollado - particularmente en México- a través de estrategias de estabilización macroeconómica, privatización del sector público, apertura de la economía nacional, redefinición y adecuación de la regulación económica, fueron defendidas fervorosamente por las nuevas élites políticas y tecnocráticas que se posicionaron en espacios estratégicos del sistema político de estas naciones, y que se insertan de manera activa a las redes transnacionalizadas de funcionarios que comparten similares intereses y supuestos teóricos, éticos y normativos.

En América Latina el empresariado nacional -especialmente el relacionado con las redes empresariales globales y no así el empresariado industrial subsidiado por el Estado-se benefició con los ajustes fiscales, el abatimiento de la inflación, la flexibilización del tipo de cambio y la remoción de las barreras arancelarias, pues ello facilitó la reinserción de las empresas y plataformas exportadoras en los encadenamientos mercantiles mundiales. Además, el desmonte y privatización del sector público inauguraron inéditos espacios y fuentes de acumulación de capital en sectores económicos estratégicos anteriormente regulados y bajo custodia del Estado.

No menos importante en los esfuerzos transterritoriales por crear "propicios entornos institucionales" para la expansión e integración global del capitalismo, fue la erosión de la era del desarrollo -con su consustancial política de industrialización y demás mecanismos estatales para articular el mercado interno-y la ruptura sistemática del pacto desarrollista que reivindicó derechos fundamentales de los trabajadores y que intentó modificar las relaciones entre el capital, el Estado y la fuerza de trabajo. Si bien, ciertos grupos del empresariado local/nacional fueron una estación de paso en el despliegue y afianzamiento de las redes empresariales globales, y en otros más se condenó a la quiebra, absorción y extinción a través de los procesos de fusiones y adquisiciones de empresas y bancos, en general las élites económicas y políticas compenetradas con las concepciones y estrategias del nuevo modelo económico, fortalecieron 
-desde principios de la década de los noventa y en detrimento de las potestades y alcances del Estado- su poder e influencia ideológica en las sociedades, así como su posicionamiento conservador en torno a la agenda pública (algunos estudios se observan en Babb, 2001; Camp, 2012; Salas-Porras, 2014; Enríquez Pérez, 2018).

Esta nueva correlación de fuerzas entre el Estado y el mercado se fundamentó en el carácter incisivo para denostar y sobredimensionar los fallos y rigidez organizacional del primero, así como en la entronización de la iniciativa privada bajo la creencia de la eficiencia económica como mecanismo principal para la asignación de recursos y para la redistribución de la riqueza. Fue un viraje que subsumió los esfuerzos de la acción colectiva desde el sector público a la lógica del fundamentalismo de mercado y a la ideología del individualismo a ultranza. $\mathrm{Y}$ en ello, las élites políticas tecnocráticas con orientación empresarial encontraron el terreno fértil para desplegar su poder y ejercicio tanto en los centros decisorios del Estado como en la empresa privada (la llamada puerta giratoria); lo que influyó en la depredación institucional y -para el caso de México- en la configuración, desde la década de los ochenta hasta el año 2018, de una red de poder transexenal (sobre este argumento véase Salas-Porras, 2014).

Respecto a una agenda social orientada al combate de la pobreza logramos también rastrear la mencionada teledirección en los esfuerzos de intervención política y disciplinamiento de las sociedades receptoras de asistencia y Ayuda Oficial para el Desarrollo. Si bien los lineamientos ideológicos y los objetivos políticos eran diferentes, desde el lanzamiento de la Alianza para el Progreso en 1961, existe una presencia constante y un interés de los organismos internacionales y de las agencias de cooperación para el desarrollo en los procesos de planeación en América Latina. En el contexto histórico de la "política de contención", en aras de enfrentar la amenaza ideológica del bloque soviético y de la Revolución Cubana, la estrategia de cuidar el "patio trasero" fue adoptada y promovida por los Estados Unidos con la fundación de la Organización de Estados Americanos (OEA) en 1958, del Banco Interamericano de Desarrollo (BID) en 1959, y de la United States Agency for International Development (USAID) en 1961. Se trataba de los años esplendorosos de la era del desarrollo; y con la Carta de Punta del Este se canalizaron 20000 millones de dólares con el fin de mejorar las condiciones sociales y económicas de las naciones latinoamericanas, a cambio de adoptar reformas estructurales, respetar el destino de la ayuda, y otorgarle un giro ingenieril al tratamiento de la pobreza tras impulsar la tecnificación de las agencias estatales y la adopción del proceso de planeación para racionalizar las decisiones y los mecanismos de intervención que contribuyesen a crear las "condiciones previas para el desarrollo" [en México, por ejemplo, el gobierno federal respondió con el llamado Plan de Acción Inmediata 1962-1964 
(Comisión Intersecretarial, 1962)]. Sin embargo, estas aristas técnicas del desarrollo no son políticamente neutrales, pese a que así lo declaren los líderes de los organismos internacionales. En el fondo de este tratamiento, subyacía la noción de que los patrones, valores, tradiciones y prácticas culturales de las naciones atrasadas obstaculizan su desarrollo al inhibir la participación social, y que la ayuda oficial sería destinada a la construcción de infraestructura y servicios que permitiesen el acceso de los pobres a los satisfactores de necesidades.

La OEA, por ejemplo, en el tratamiento de la pobreza durante las décadas de los sesenta y setenta adoptó la técnica social del "desarrollo de la comunidad" (desplegar la planeación "desde la base" e incentivar la participación de la población con el apoyo técnico del promotor, el experto y el trabajador social en la modificación educativo/pedagógica de las actitudes y psicología de sus beneficiarios en aras de impulsar el "take-off" de los pobres; y centraren ello el proceso de cambio), teorizada y confeccionada por el especialista argentino Ezequiel Ander-Egg (2002). Se trató de una técnica influyente en el diseño y ejercicio de la política social adoptada por los gobiernos latinoamericanos a partir de la puesta en marcha de los preceptos normativos y estrategias de la Alianza para el Progreso, hasta el abandono de las estrategias desarrollistas en la década de los ochenta.

Por su parte, con la estrategia para la satisfacción de las necesidades básicas (un primer precedente puede rastrearse en el llamado Informe Pearson) adoptada a principios de la década de los setenta, el Banco Mundial también atendió el problema de la pobreza al relacionar -bajo la óptica de Robert S. McNamaralas posibilidades de desarrollo, con la seguridad nacional y la estabilidad sociopolítica, que tienen como finalidad el procurar la cobertura de servicios básicos no materiales para enfrentar la desigualdad en la distribución del ingreso y la provisión de alimentos al interior de los hogares. Esta estrategia del Banco Mundial fue la respuesta a la crisis estructural del capitalismo gestada en la década de los setenta, y tuvo como objetivo oficial luchar contra la pobreza y aumentar el suministro de servicios básicos en rubros como el control demográfico, la educación y la salud en los países subdesarrollados (véase World Bank, 1975), y sentar así las bases -frente al recrudecimiento de la guerrilla urbana-para la procuración de una seguridad política interna que facilitase la adopción de procesos de ajuste estructural en los sectores manufacturero y exportador, de cara a la eficaz inserción productiva internacional de los países que, relativamente, lograron industrializarse en las décadas previas (véase también Streeten, 1986)

En algunas naciones subdesarrolladas, como México, el mismo Banco Mundial - hacia principios de la década de los setenta- promovió la estrategia del desarrollo rural integrado como el camino para superar la pobreza en el campo 
a través de la llamada "Revolución Verde" y de su proceso de tecnificación de la producción agrícola. El objetivo consistía en incrementar la productividad en el campo para que los pobres viesen aumentados sus ingresos per cápita. Al respecto, se asumió como crucial el canalizar la inversión pública a la creación de mecanismos que proveyesen a los pobres de financiamiento, insumos, información, infraestructuras, conocimientos técnicos y tecnología, así como de tierra apta para el cultivo (sobre esta estrategia del Banco Mundial consúltese Chenery, Ahluwalia, Bell, Duloy y Jolly, 1976).

En particular, desde mediados de la década de los noventa y tras las inconsistencias y fracasos de las políticas de ajuste y cambio estructural adoptadas durante los años ochenta y tras la oleada del fundamentalismo de mercado, varios organismos internacionales retomaron el interés por el tema de la pobreza. Como parte de la división técnica del trabajo se entrelazaron de una forma más consistente las funciones de entidades como el Fondo Monetario Internacional (FMI), el Banco Mundial, organismos específicos del Sistema de la Organización de las Naciones Unidas como el PNUD y agencias de donantes bilaterales, para lograr la correspondencia entre las donaciones y préstamos subsidiados (la ayuda oficial y la asistencia concesionaria) de los dos organismos financieros referidos, y la llamada Iniciativa Reforzada para los Países Pobres Muy Endeudados, con la finalidad de que las sociedades de bajos ingresos adoptaran estrategias nacionales de desarrollo y de lucha contra la pobreza sin salirse del cauce mismo de las directrices del Consenso de Washington, custodiadas celosamente por el FMI y apuntaladas desde 1997 con la segunda generación de reformas. Estas estrategias serían expuestas por parte de los gobiernos nacionales en los llamados Documentos de Estrategia de Lucha Contra la Pobreza (DELP), que serían integrados en las políticas públicas ejercidas en esos países. La teledirección en el ámbito de la política social se manifiesta también en las directrices estipuladas en la Declaración del Milenio (Naciones Unidas, 2000), en los Objetivos de Desarrollo del Milenio -que regirían los criterios y prácticas de la cooperación internacional entre el año 2000 y el 2015-, y la Agenda 2030 para el Desarrollo Sostenible adoptada desde septiembre de 2015, y en los cuales concurren múltiples organismos internacionales (Naciones Unidas/ Asamblea General, 2015).

A grandes rasgos, los espacios globales para la toma de decisiones y, especialmente, los organismos internacionales, se erigen en estructuras políticas cuyas funciones se engarzan en una sofisticada y sistemática división técnica del trabajo relativa a la cooperación internacional y que -a través de la teledirección - inciden, como parte de una lógica multidireccional, en la toma de decisiones y en la configuración de la agenda pública nacional de los Estados subdesarrollados que necesitan de sus mecanismos y servicios de capacitación 
y de asistencia técnico/financiera. De ahí que, en correspondencia a sus funciones políticas, inciden en la constelación de principios jurídicos y de regímenes internacionales que regulan las interacciones entre el mercado y los Estados, así como en los mecanismos de negociación de las estructuras de poder mundial y en la perpetuación de las asimetrías propias de las relaciones económicas y políticas interestatales.

\section{La crisis de Estado en México y la inserción desventajosa en los espacios globales para la toma de decisiones}

Si buena parte de las decisiones en materia de política pública y de modelación de la dialéctica desarrollo/subdesarrollo no se toman exclusivamente en las escalas nacionales y en las agencias de planeación de las sociedades subdesarrolladas, cabe puntualizar que en ello intervienen múltiples fuerzas, actores $\mathrm{y}$ agentes transnacionalizados que conforman cuerpos de alta especialidad y provistos de cohesión y sincronización, que integran a las élites tecnocráticas nacionales que se identifican y mantienen compromisos estrechos con dichas comunidades epistémicas transterritoriales.

Sin embargo, en estos espacios globales para la toma de decisiones, los Estados se insertan de manera diferenciada y asimétrica, y con mayor razón cuando experimentan una erosión, debilidad y fragilidad de sus instituciones fundamentales. Esta crisis de Estado experimentada en naciones como México responde a la gravitación desplegada - desde afuera y desde adentro, desde arriba y desde abajo- por múltiples fuerzas, factores y poderes fácticos que inciden en la configuración de las decisiones públicas y que, con ello, constriñen los alcances y profundizan las limitaciones de las instituciones estatales.

Entre estas fuerzas gravitatorias destacan los organismos internacionales y las redes que tejen con otros agentes en torno a la definición de los problemas públicos; al estudio y tratamiento de los mismos; y al delineamiento de directrices de política. Aunque se forma una élite transnacional que perfila decisiones públicas para confeccionar soluciones locales/nacionales respecto a problemáticas que tienen resortes globales, no todos los Estados, agentes y funcionariados participan en igualdad de circunstancias en esos espacios globales para la toma de decisiones; y ello se relaciona, en primera instancia, con la posición de las sociedades y los gobiernos nacionales en la estratificada economía mundial y en el curso de la disruptiva dialéctica desarrollo/subdesarrollo. 
Paralelamente, la debilidad y depredación de los entramados institucionales en las sociedades subdesarrolladas contribuye a esta inserción desventajosa, a medida que los Estados son socavados en las potestades que les dieron sentido histórico, pierden protagonismo, y es trastocada y vaciada de contenido su soberanía.

Entre estas fuerzas y poderes fácticos que asedian a un Estado subdesarrollado como el mexicano y que inciden en su agenda pública, destacan: a) desde afuera, la intensificación de los procesos de globalización; la irradiación del mercado global; la apertura comercial y los sistemas internacionales de producción integrada; los procesos de integración económica y regionalización; los mismos organismos interestatales y sus consustanciales regímenes internacionales; las redes globales de toma de decisiones; las comunidades epistémicas y las élites financieras y tecnocráticas transnacionales, b) desde adentro, el crimen organizado; los grupos sociales que controlan extensas franjas del territorio nacional y que le disputan al Estado el monopolio legítimo de la violencia, c) desde arriba, el empresariado privado; los inversionistas institucionales y los especuladores financieros; los líderes de opinión y los medios masivos de difusión; las iglesias y las múltiples teologías; los líderes gremiales y sindicales; las élites políticas corruptas, rentistas y patrimonialistas que se apropian de lo público, y d) desde abajo, los grupos de presión; los paramilitares; los movimientos guerrilleros; las organizaciones de base; las comunidades que toman justicia por propia mano.

Ante la proliferación de estas fuerzas y poderes fácticos que sitian y desbordan al Estado subdesarrollado y le disputan su hegemonía, la soberanía nacional -en tanto principio jurídico/político fundamentado en la autodeterminación y en la capacidad para tomar decisiones estratégicas- se difumina y se comparte con concepciones e intereses transnacionales como los representados en los espacios globales para la toma de decisiones.

Si las élites políticas nacionales y las instituciones estatales ven erosionadas su capacidad y potestades para articular, regular y cohesionar a sus sociedades, en el contexto de un cambiante y volátil equilibrio de fuerzas, las decisiones públicas se exponen a los imperativos de estas fuerzas, factores y poderes fácticos. Paralelamente a ello -o, incluso, como parte de ello- el agotamiento y desmonte del Estado desarrollista se traslapó con la reconfiguración de las élites políticas y con la relativa pérdida de control sobre el territorio, los poderes fácticos anteriormente referidos, y sobre las posibilidades de modelación de la sociedad y su correspondiente dialéctica desarrollo/subdesarrollo. Cabe destacar que a lo largo del siglo XX mexicano, el Estado y su partido cuasi oficial, relativamente, lograron controlar y cohesionar a algunos de esos poderes fácticos; situación 
que cambió desde el año 2000 con la primera alternancia partidista y continúa cambiando hasta la fecha.

Esta crisis de Estado -signada por la debilidad institucional y la proliferación y expansión de poderes fácticos- está en función de las transformaciones finiseculares del Estado y de sus funciones esenciales relacionadas con la construcción de mercados, la redistribución de la riqueza y la protección de los derechos humanos básicos. Más aún, responde a procesos de largo alcance histórico relacionados con el desprestigio, subsunción, erosión y volatilización de la política como praxis que dota de posibilidades para resolver los problemas públicos, impulsar transformaciones sociales de gran calado, y para generar esperanzas entre los individuos y las sociedades. Esta pérdida de fe y confianza en la política y el Estado abona el terreno para que los poderes fácticos se posicionen en la agenda pública y en las decisiones discrecionales de las élites políticas; al extremo de borrarse los límites entre lo público y lo privado.

Particularmente, los actores socioeconómicos y políticos que participan en las comunidades epistémicas transterritoriales y en los espacios globales para la toma de decisiones no siempre responden a objetivos e intereses plenamente públicos. En el caso de México, al ser élites hasta cierto punto desarraigadas de las necesidades y problemáticas de las sociedades subdesarrolladas nacionales y al poseer concepciones etnocéntricas y sujetas a la teoría económica neoclásica, subsumen otros saberes y formas de representar la realidad (Pedro Aspe, Ministro de Haciendo entre 1988 y 1994 declaró que "la pobreza es un mito genial"; Ernesto Cordero, otro Ministro de Hacienda, declaró en 2011 que “... hay familias mexicanas que con ingresos de seis mil pesos al mes tienen crédito para una vivienda, tienen crédito para un coche, se dan tiempo de mandar a sus hijos a una escuela privada y están pagando las colegiaturas").

Más todavía, si las deliberaciones se toman a puerta cerrada y con representantes de un Estado sitiado y en condiciones de debilidad institucional, no es de extrañar que las decisiones tomadas resulten antidemocráticas y antipopulares ante el predominio de los intereses creados, privados o de grupo. Ante estas estructuras transnacionales de poder dotadas de amplias funciones epistémico/cognitivas, pese a que pertenecer a ellas es del interés genuino y de pleno convencimiento de las élites políticas de orientación tecnocrática, el Estado mexicano se inserta de manera desventajosa y subordinada en el proceso de toma de decisiones.

Esto se observa, por ejemplo, en las recientes negociaciones orientadas a reformar (o, en su defecto, a suprimir) el Tratado de Libre Comercio de América del Norte (TLCAN), donde la delegación mexicana está dispuesta a ceder al 
extremo con tal de evitar la retirada de las inversiones extranjeras que sostienen el raquítico crecimiento económico. Más en un terreno fértil dado por la incapacidad para contener los flujos migratorios hacia Estados Unidos y arraigarlos en sus lugares de origen, así como por la falta de control del Estado sobre el territorio y el crimen organizado que alcanza ramificaciones transnacionales.

\section{Conclusiones}

La definición de los problemas públicos y el proceso de toma de decisiones relativo a la planeación y a la intervención del Estado en los cauces de la dialéctica desarrollo/subdesarrollo está en función de la estructuración de constelaciones de poder que trascienden las fronteras nacionales y que al insertarse en ellas, las élites políticas tienden a reconfigurarse y a compenetrarse decididamente con aquellas concepciones de la realidad social y del proceso económico que no trastocan los fundamentos y la lógica de la expansión e integración global del capitalismo. Más bien, con esa inserción -en múltiples casos desventajosa y en condiciones de debilidad institucional para los Estados subdesarrolladosestas minorías poderosas contribuyen a apuntalar los procesos de acumulación y los mecanismos de legitimidad que son indispensables en el despliegue de la economía mundial y la política internacional.

Por último, cabe destacar que la gravitación y predominio de las comunidades epistémicas transterritoriales y de los espacios globales para la toma de decisiones en la construcción de la agenda pública y de las estrategias de desarrollo se correlaciona con la crisis de Estado experimentada en México desde hace dos décadas y media, y ello nos convoca a la necesidad de reflexionar y analizar en torno a la lógica y el sentido que adopta la toma de decisiones públicas en materia de planeación del desarrollo; el papel del poder epistémico/cognitivo de los organismos internacionales donde los funcionariados mexicanos asumen un papel activo; y la reconfiguración de las élites políticas nacionales de cara a la convergencia, estandarización, sincronización, armonización, homogeneización y coordinación de las políticas públicas cada vez más transnacionalizadas.

Si bien el poder epistémico/cognitivo de los organismos internacionales es diferenciado y dista de ser homogéneo entre ellos, su incidencia en las escalas nacionales de la planeación está en función de la agenda pública de los gobiernos y de las concepciones sobre el desarrollo que en ellos predomina. No menos importante es el perfil, formación y comportamiento de las élites políticas nacionales que se insertan en los espacios globales para la toma de decisiones. Ello nos obliga a explorar en futuras investigaciones las posibles contribuciones de las ciencias sociales del sur del mundo, los saberes periféricos y su pensamiento 
social crítico, en aras de reconstruir el debate en torno a la configuración de la agenda pública. De ahí que resulte preciso articular un discurso teórico alternativo con sus respectivas pautas de política pública para posicionarlo en el seno de las comunidades epistémicas transterritoriales y en la agenda de los organismos internacionales. No sin antes pensar en la necesidad de perfilar los fundamentos de una posible sociología política o una economía política de los organismos internacionales y de la relación orgánica de estos con los gobiernos de Estados subdesarrollados. Más urgentes resultan estas necesidades teóricas de cara a la crisis de sentido que invade a las sociedades contemporáneas, y ante la generalizada desconfianza en la praxis política, la vida pública y la acción colectiva; tan indispensables en la reconstrucción de proyectos alternativos de Nación acordes a los problemas públicos y a las necesidades del mundo subdesarrollado.

\section{Referencias}

Ander-Egg, Ezequiel. (2002). Metodología y práctica del desarrollo de la comunidad. (Tomo I, II y III), Buenos Aires, Editorial Lumen, Trigésimo Tercera Edición.

Ascolani, Adrián. (2008). "Estrategias del Banco Mundial para el financiamiento de la educación en los países latinoamericanos", en: revista Educação. Porto Alegre, vol. 31, núm. 2, maio/ago, pp. 139-156.

Babb, Sarah L. (2001). Managing Mexico: economists from nationalism to neoliberalism, Princeton, Princeton University Press.

(2009). Behind the Development Banks: Washington politics, world poverty, and the wealthy of nations. Chicago and London, The University of Chicago Press.

Banco Mundial. (2014). "Un modelo de México para el mundo", Comunicado de Prensa, Banco Mundial, alojado en: http://www.bancomundial.org/es/news/ feature/2014/11/19/un-modelo-de-mexico-para-el-mundo

Barrera Cordero, Juan. (2009). "La guerra del agua en Cochabamba: un caso de palabras que hablan mal”, en: Investigación ambiental. Ciencia y politica pública. México, Instituto Nacional de Ecología y Cambio Climático, núm. 1, pp. 91-100.

Burgos Silva, Germán. (2009). Estado de derecho y globalización: el Banco Mundial y las reformas institucionales en América Latina. Bogotá, ILSA y Universidad Nacional de Colombia. 
. (2015). "El Banco Mundial y los derechos humanos: un repaso crítico a los argumentos jurídico-doctrinales", en: Revista de Relaciones Internacionales, Estrategia y Seguridad. Bogotá, Universidad Militar Nueva Granada, vol. 10, núm. 1, pp. 245-265.

Burki, Shahid Javed y Guillermo E. Perry. (1998). Más allá del consenso de Washington. La hora de la reforma institucional. Washington, Banco Mundial.

Camp, Roderic Ai. (2012). Metamorfosis del liderazgo en el México democrático. México, Fondo de Cultura Económica (FCE), (1ra. Edición en Inglés: 2010).

Chenery, Hollis, Montek S. Ahluwalia, C. L. G. Bell, John H. Duloy y Richard Jolly. (1976). Redistribución con crecimiento. Políticas para mejorar la distribución del ingreso en los países en desarrollo en el contexto del crecimiento económico. Madrid, Editorial Tecnos, (1ra. Edición en Inglés: 1974).

Coase, Ronald H. (1984). "The new institutional economics", in: Journal of Theoretical and Institutional Economics, vol. 140, number 1, pp. 229-231.

Comisión Intersecretarial. (1985). "Plan de Acción Inmediata 1962-1964”, en: Antología de la planeación en México (1917-1985). Los programas de desarrollo y la inversión pública (1958-1970), volumen III, México, Secretaría de Programación y Presupuesto y FCE (1ra. Edición: 1962), pp. 23-70.

Corbalán, María Alejandra. (2002). El Banco mundial, intervención y disciplinamiento. El caso argentino, enseñanzas para América Latina. Buenos Aires, Ed. Biblos.

Cox, Robert W. (1981). "Social forces, States and world orders: beyond international relations theory", in: Millennium: Journal of International Studies, vol. 10, number 2, June, pages 126-155.

. (1983). "Gramsci, hegemony and international relations: an essay in method”, in: Millennium: Journal of International Studies, vol. 12, number. 2, pages 162-175.

. (1987). Production, power and world order: social forces in the making of history. Nueva York, Columbia University Press.

and Michael G. Schechter. (2002). Political economy of a plural world: Critical reflections on power, morals and civilization. London, Routledge.

Delgado-Ramos, Gian Carlo y John Saxe-Fernández. (2005). “The World Bank and the privatization of public education: a mexican perspective, in: Journal for Critical Education Policy Studies, vol. 3, number 1, 33 pages.

Enríquez Pérez, Isaac. (2009). "La evolución de las concepciones sobre el desarrollo en el Banco Mundial y su incidencia en América latina: un panorama general", en: Revista Nicolaita de Estudios Económicos. Morelia, Universi- 
dad Michoacana de San Nicolás de Hidalgo, Vol. IV. Núm. 1, enero-junio, pp. 111-140.

. (2010). "La evolución del pensamiento sobre el desarrollo en el Fondo Monetario Internacional y el Banco Mundial: un análisis comparativo sobre su gravitación en América Latina”, en: Trayectorias. Revista de ciencias sociales. Monterrey, Universidad Autónoma de Nuevo León, Vol. XII, núm. 31, pp. 31-60.

. (2014). "La perspectiva del desarrollo sostenible en el pensamiento del Banco Mundial: la asimétrica simbiosis entre la agenda económica y la agenda ambiental", en: Realidad económica. Revista de ciencias sociales. Buenos Aires, Instituto Argentino para el Desarrollo Económico, núm. 287, pp. 118140 .

. (2015). Los organismos internacionales y su incidencia en el desarrollo latinoamericano: la gravitación del pensamiento y las estrategias del Banco Mundial en las políticas públicas mexicanas. Madrid, Universidad Complutense de Madrid. Alojada en: http://eprints.ucm.es/33678/1/T36554.pdf

. (2016a). Las estrategias de desarrollo y los avatares de la planeación nacional: un estudio sociohistórico para la reconstrucción de un paradigma perdido en las políticas públicas mexicanas, Saarbrücken, Editorial Dictus.

. (2016b). "Los senderos de los organismos internacionales en la cooperación para el desarrollo: un panorama general sobre la evolución de la estrategias del Banco Mundial”, en: revista Análisis político. Bogotá, Universidad Nacional de Colombia, núm. 88, diciembre, pp. 105-125.

. (2017). "Los organismos internacionales y su incidencia en la dialéctica desarrollo/subdesarrollo: notas introductorias para comprender su naturaleza, funciones y comportamiento", en: Revista de Relaciones Internacionales de la UNAM, núm. 127, pp. 49-81.

. (2018). "La incidencia del factor empresarial en la dialéctica desarrollo/ subdesarrollo: las racionalidades empresariales y el capital social en los espacios locales/regionales", en: ICADE. Revista de las Facultades de Derecho y Ciencias Económicas y Empresariales. Madrid, Universidad Pontificia Comillas, núm. 104, mayo-agosto.

Fal, Juan. (2013). "Las operaciones del Banco Mundial en la Argentina: injerencia en el sector agrícola. Notas sobre el control territorial, social y biológico", en: Periferias. Revista de Ciencias Sociales. Buenos Aires, Fundación de Investigaciones Sociales y Políticas, número 21, primer semestre, pp. 61-87.

Haas, Peter M. (Editor). (1992). "Knowledge, power and international policy coordination", in: International Organization Review, vol. 46, n. 1, winter. 
International Monetary Fund and World Bank. (1999). Formulación de estrategias de reducción de la pobreza en los países en desarrollo. Washington, International Monetary Fund and World Bank.

Keohane, Robert O. (1989). International Institutions and State Power. Essays in International Relations Theory, Boulder, Westview Press. . (1998). "International Institutions: Can Interdependence Work?" Foreign Policy, number 110, Spring, pages 82-96.

Levy, Santiago. (2008). Good intentions, bad outcomes: social policy, informality, and economic growth in Mexico. Washington, D. C., Brookings Institution Press.

Naciones Unidas (Asamblea General). (2000). Declaración del milenio, Quincuagésimo Periodo de Sesiones el 8 de septiembre de 2000, Nueva York, Naciones Unidas.

. (2015). Transformar nuestro mundo: la Agenda 2030 para el Desarrollo Sostenible, Resolución aprobada por la Asamblea General el 1 de septiembre de 2015, Sexagésimo noveno período de sesiones, Nueva York, Naciones Unidas.

Noy, Shiri. (2013). "Las políticas de salud del Banco Mundial”, en: Revista de Ciencias Sociales, San José, Universidad de Costa Rica, núm. 142, pp. 75-85.

Picazzo Palencia, Esteban. (2012). Acceso a la salud, equidad y sustentabilidad: caso aplicado para el estado de Nuevo León. Monterrey, Tesis Doctoral presentada en el Doctorado en Ciencias Sociales, Universidad Autónoma de Nuevo León.

Putnam, Robert D. (1993). Making democracy work. Civic traditions in modern Italy. Princeton, Princeton University Press.

Reyes Cabrera, Edilberto. (2015). Políticas de educación superior en México 19942003: Recomendaciones e influencia de los organismos internacionales, México, Tesina para obtener el Título de Licenciado en Ciencia Política, UAM-Unidad Iztapalapa, 109 pp.

Salas-Porras, Alejandra. (2014). "Las élites neoliberales en México: ¿Cómo se construye un campo de poder que transforma las prácticas sociales de las élites políticas?”, en: Revista Mexicana de Ciencias Políticas y Sociales, UNAM. Núm. 222, 279-312.

Sachs, Wolfgang (Editor). (1992). The development dictionary: a guide to knowledge as power, New York, Zed Books.

Sánchez Cerón, Manuel. (2001). "Influencia del Banco Mundial y la CEPAL en las tendencias educativas recientes en algunos países latinoamericanos", en: 
Revista Latinoamericana de Estudios Educativos. México, vol. 31, núm. 4, pp. 55-97.

Santiso, Carlos. (2001). “Gobernabilidad democrática y reformas económicas de segunda generación en América Latina”, en: Revista Instituciones y Desarrollo. Barcelona, Institut Internacional de Governabilitat de Catalunya, núm, 8 y 9 , pp. 325-366

. (2004). "The contentious Washington Consensus: reforming the reforms in emerging markets", in: Review of International Political Economy, vol. 11, number 4, pp. 828-844.

Saxe-Fernández, John y Gian Carlo Delgado-Ramos. (2003). Banco Mundial y desnacionalización integral en México. México, UNAM.

. (2004). Imperialismo y Banco Mundial. Madrid, Editorial Popular.

. (2005). Imperialismo económico en México. Las operaciones del Banco Mundial en nuestro país. México, Editorial Debate.

Sen, Amartya K. (1999). Development as freedom, New York, Oxford University Press.

Streeten, Paul, Shahid Javed Burki, Mahbub ul Haq, Norman Hicks y Frances Stewart (1986), Primero lo primero: satisfacer las necesidades humanas básicas en los países en desarrollo, Madrid, Editorial Tecnos (1ra. Edición en Inglés: 1981).

Toussaint, Eric. (2006). Banco Mundial, el golpe de estado permanente. La agenda oculta del Consenso de Washington. España, El viejo topo.

Valdez, José Fernando. (2015). El gobierno de las élites globales. Cómo se organiza el consentimiento, La experiencia del Triángulo Norte. Guatemala, Instituto de Investigaciones y Gerencia Política, Cara Parens y Universidad Rafael Landívar.

Williamson, Oliver E. (1985). The economics institutions of capitalism: firms, markets, relational contracting. Nueva York, The Free Press.

. (1995). "The institutions and governance of economic development and reform", Proceedings of the World Bank Annual Conference on Development Economics 1994. Washington, World Bank.

. (1996). The mechanisms of governance. New York, Oxford University Press.

. (2000). "The new institutional economics: taking stock, looking ahead", in: Journal of Economic Literature, American Economic Association, vol. XXXVIII, number 3, pp. 595-613. 
World Bank. (1975). The assault on world poverty. Problems of rural development education and health. Baltimore, The Johns Hopkins University Press. . (1990). World Development Report 1990. Poverty, Oxford, Oxford University Press. . (2001). Informe sobre el desarrollo mundial 2000-2001. Lucha contra la pobreza. Washington, Mundi-Prensa Libros, (1ra. Edición en Inglés: 2000). 
